\title{
Ovarian cancer G protein-coupled receptor 1 inhib- its A549 cells Migration through Casein kinase $2 \alpha$ intronless gene
}

Adhikarimayum Lakhikumar Sharma ${ }^{1,2}$, Puyam Milan Meitei ${ }^{1}$, Naorem Tarundas Singh ${ }^{1}$, Thiyam Ramsing Singh ${ }^{1}$, Lisam Shanjukumar Singh ${ }^{1 *}$

${ }^{1}$ Cancer and Molecular Biology Division, Department of Biotechnology, Manipur University, Canchipur- 795003, Manipur, India

${ }^{2}$ Current Address: Center for Translational Medicine, Thomas Jefferson University, 1020 Locust Street, Philadelphia, PA, USA

* Corresponding Author: Correspondence to Lisam Shanjukumar Singh, Cancer and Molecular Biology Division, Department of Biotechnology, Manipur University, Canchipur-795003, Manipur, India,

Tel: +91 9615533300; fax: +91 385 2435145; e-mail: shanju.lisam@manipuruniv.ac.in

\section{Abstract}

We have previously reported that ovarian cancer G protein-coupled receptor 1 (OGR1) is a new metastasis suppressor gene. We have also reported for the first time that a new intronless gene for casein kinase $2 \alpha$ (CSNK2A3) is expressed in human cells. The promoter of the well-known casein kinase $2 \alpha$ (CSNK2A1) displays characteristics of housekeeping gene whereas CSNK2A3 has a characteristic of a regulated promoter with two TATA boxes and a CAAT box. In this study, we found that OGR1 upregulates expression of CSNK2A3 by about 3 folds in A549 cells but not CSNK2A1. OGR1 also upregulates expression of neutral endopeptidase (NEP). The OGR1 induced inhibition of A549 cell migration is completely abrogated by inhibition of casein kinase $2 \alpha$ activity, whereas partial abrogation ( $30 \%$ ) was observed in the presence of NEP inhibition. The results also revealed that OGR1 regulates CSNK2A3 via activation of Rac1/cdc42 and MAPKs pathways. CK2 is ubiquitously expressed and in contrast it is believed to be a constitutively active enzyme and its regulation appears to be independent of known second messengers. There is no previous report on how expression of CK2 $\alpha$ in cancer cells is regulated although many studies have report of aberrant expression of the kinase in cancer. In the current study, we are reporting for the first time the regulation of intronless casein kinase $2 \alpha$ gene, CSNK2A3 in cancer cells. Our findings suggest that the aberrantly casein kinase $2 \alpha$ expression found in various cancer cells may the due to CSNK2A3 expression which is potentially regulated by several master regulators of the developmental pathways rather than well-known casein kinase $2 \alpha$ gene, CSNK2A1.

Key words: ovarian cancer G protein-coupled receptor 1; casein kinase $2 \alpha$; intronless; neutral endopeptidase

Running title: OGR1 inhibits A549 cells Migration 


\section{Introduction}

The ovarian cancer G protein-coupled receptor 1 (OGR1) and related subfamily members mediate the functions of several lysophospholipids which include endothelial barrier function, endothelial cell proliferation, migration, and tube formation, T cell migration, glucocorticoid-induced thymocyte apoptosis, and globoid cell formation [1-5]. OGR1 gene has been shown to be expressed at lower levels in metastatic compared with primary prostate cancer tissues [6]. Recently, we have shown using an orthotopic mouse metastasis model that OGR1 is a novel metastasis suppressor gene for prostate cancer when it is re-expressed in cancer cells [5]. Further, other researchers have also reported that OGR1 inhibits breast and ovarian cancer cells in vitro when it is re-expressed in cancer cells [7, 8]. However, our previous study revealed that OGR1 has inhibitory effect on prostate cancer tumorigenesis when expressed in host/stromal cells using OGR1 knockout TRAMP mice prostate cancer model [9]. Further, we have also demonstrated that OGR1 significantly inhibited melanoma tumorigenesis in OGR1 knockout mice [10]. Another study of Horman SR, et al. has reported that murine colon tumor implants in OGR1 knockout mice displayed delayed tumor growth [11]. Therefore, these previous reports clearly indicate that in contrast to OGR1's tumor-suppressing role in tumor cells, host cell OGR1 may be involved in and/or required for tumor growth. OGR1 and its subfamily G protein-coupled receptors (GPCRs), GPR4, G2A and T-cell death-associated gene 8 (TDAG8), have been shown to have protonsensing ability $[12,13]$. Many of these proton sensing activities have been identified in cells overexpressing one or more of these GPCRs. More recently, proton sensing activities have been detected in cells from GPR4- and TDAG8-, but not G2A-deficient mice [3, 14, 15]. Previous studies have reported that OGR1 activity on tumorigenesis is independent of its $\mathrm{pH}$-sensing activities. These G-protein coupled receptors also appear to have ligand-independent constitutive activity $[5,7,8,10]$. However, the signalling pathway of OGR1 in inhibition of metastasis is not clearly understood. One previous report has revealed that OGR1 induced activation of Rho but down-regulation of Rac1 in breast cancer cell line [8]. Therefore, it is important to investigate the downstream cellular proteins of OGR1 in its function as metastasis suppressor gene.

Casein kinase $2 \alpha(\mathrm{CK} 2 \alpha)$ acts as a regulator of several hallmarks of cancer cell behaviour [16-18]. CK2 $\alpha$ gene may potentially be induced or repressed by several master regulators of developmental pathways [19-21]. We have for the first time reported that an intronless gene of CK2 $\alpha$ (CSNK2A3) is expressed in human megakaryocytic cells [21]. Recently other studies have revealed that CSNK2A3 is expressed in 293T, A549 and NIH-3T3 cells and further polymorphism of CSNK2A3 plays oncogenic roles in lung cancer [22]. Importantly, although the activity of the CSNK2A1 gene has been shown to be elevated in human cancers, no solid genetic or epigenetic evidence is available regarding the cause of the high-activity of CK2 $\alpha$ in cancer cells. CK2 proteins are upregulated in the human tumors tested so far, suggesting its important role in cancer progression [23, 24]. In cancer, CK2 is proposed to regulate essential cellular processes such as cell growth [25], cell proliferation [26], cell survival [27], cell morphology [28], cell transformation [29,30] and angiogenesis [31]. Although, it is observed that 
there are multiple layers of regulation of CK2 $\alpha$ expression [24, 32], reports on regulation of CK2 $\alpha$ expression is very limited. The original view in the literature is that CK2 is predominantly regulated posttranscriptionally; however, recent studies strongly suggest that regulation at the transcriptional level is also important in some cancers [33]. Recently, Das, $\mathrm{N}$ et al have reported that ER $\alpha$ transcriptionally activates CKa [34]. Unpredictably, some cancers show under-expression of CK2 transcripts in breast, ovarian, and pancreatic cancer [33]. Furthermore, CK2 transcript levels could have a prognostic value in cancers (e.g. CK2 $\alpha$ in squamous cell carcinoma of the lung). For the most part, high levels of CK2 transcript correlate with lower overall survival (e.g. breast and ovarian cancer, glioblastoma, kidney and liver cancer) [33, 35-37]. However, in lung adenocarcinoma, high levels of CSNK2A2 (CK2 $\left.\alpha^{\prime}\right)$ and CSNK2A3 correlate with higher survival rates [24, 33]. Similar to lung adenocarcinoma, overexpression of CSNK2A3 in renal clear cell carcinoma led to increased survival [24]. From the above data, it is not clear whether CK2 is anti-cancer or pro-cancer molecule.

Neutral endopeptidase 24.11 (NEP, neprilysin, enkephalinase, CD 10) is a widely distributed membrane enzyme, occurring on a variety of cells [38]. The biological and regulatory effects of NEP are presumed only to result from its enzymatic function [39, 40]. However, recent data suggest that NEP protein expression in of itself can effect signal transduction pathways that regulate cell growth [41, 42] and apoptosis [43]. Tokuhara et al reported tumors with high NEP and low CD13 were associated with better prognoses [44]. Gurel et al reported that in lung squamous cell carcinomas both tumoral and stromal NEP expression were unfavorable prognostic factors, while in non-squamous cell carcinomas tumoral NEP expression was a favorable prognostic factor [45]. In a study conducted by Kristiansen et al, tumoral NEP expression was not associated with prognosis in NSCLCs [46]. Ono et al reported that neither tumoral nor stromal NEP expression correlated with prognosis in stage I lung squamous cell carcinomas [47]. Recently, Leithner et al reported that high NEP expression, based on gene expression analysis, was associated with unfavorable prognoses in lung adenocarcinomas but not in non-adenocarcinomas [48]. However, it is unclear whether this result was based on tumoral or stromal NEP expression because localization of NEP was not confirmed via immunohistochemistry. Therefore, the prognostic value of tumoral NEP for early-stage lung adenocarcinoma remains unknown. NEP expression was lower in certain carcinomas of the lung than in adjacent normal tissue [38]. In the current study we aim to identify the key cellular protein(s) involved in the inhibition cell migratory induced by a metastasis suppressor gene, OGR1.

\section{Results}

\section{OGR1 regulates expression of CSNK2A3 and NEP but not CSNK2A1}

To investigate the downstream molecules involved in the OGR1 induced inhibition of cancer cells migration, OGR1 was over-expressed transiently in A549 cells for $48 \mathrm{hrs}$ and transcript expression of CSNK2A1, CSNK2A3 and NEP were analysed using semi-qPCR. Initially, OGR1 over-expression in A549 was confirmed by semi-qPCR. The results showed that OGR1 strongly up-regulates transcript expression of CSNK2A3 (CK2 $\alpha$ intronless gene, CK2 $\alpha \mathrm{P})$ and NEP. However, OGR1 does not affect 
significantly the expression of CSNK2A1 in A549 cells (Figure 1 A, B). Further, protein expression of CK2 $\alpha$ and NEP were analysed by immunoblotting upon OGR1 over-expression in A549 cells using respective specific antibodies (Figure 1C, D). The over-expression of OGR1 protein was confirmed using specific antibody against OGR1. The specific antibody for CK2 $\alpha$ recognises proteins of both CSNK2A1 and CSNK2A3 since there are only four amino acids different in the sequence of the two genes [21]. Taking together the results of transcript expression of casein kinase $2 \alpha$ genes (CSNK2A1 and $C S N K 2 A 3$ ) and protein expression of $\mathrm{CK} 2 \alpha$, it is clearly indicated that the increased in protein of CK2 $\alpha$ in immunoblotting may be the product of CSNK2A3 (CK2 $\alpha P)$. The immunoblotting against NEP confirmed that OGR1 over-expression cells increases NEP expression in A549 cells (Figure 1C). The overall results indicate that OGR1 up-regulates expression of CSNK2A3 and NEP, but not CSNK2A1 in lung cancer cells. Our finding is the first report to demonstrate that the expression of CSNK2A3 is regulated. There is very limited report of how $\mathrm{CK} 2 \alpha$ gene expression is regulated inspite of many reports on aberrantly expression in cancer.

\section{CK2 $2 \alpha$ is up stream of NEP in the OGR1 signalling pathway}

To investigate whether CK2 $\alpha \mathrm{P}$ is upstream of NEP or vice-versa in the OGR1 signalling pathway, we assessed expression of both the proteins in the presence of either specific chemical inhibitor of CK2, CX-4945 and immunoblotting against NEP or specific chemical inhibitor of NEP, thiophan and immunoblotting against CK2 $\alpha$ after A549 cells were transiently transfected with OGR1 or empty vector (control). Interestingly, the results showed that in the presence of CX-4945, increased expression of NEP induced by OGR1 is abrogated to a similar level of control cells (Figure 2A, B). However, thiorphan did not affect expression of CK2 $\alpha$ protein in A549 cells (Figure 2C, D). These results indicated that CK2 $\alpha P$ is upstream of NEP in the signalling pathway of OGR1. It is previously reported that CK2 $\alpha$ regulates NEP activity by phosphorylating at cytoplasmic tail [49].

Further, we have reported earlier that OGR1 inhibits PC3 cell migration via Gai activation [5]. Therefore, to investigate whether OGR1 induced up-regulation of CSNK2A3 in A549 is dependent of activation of Gai, we analysed the transcript expression of CSNK2A3 in the presence or absence of pertussis toxin (PTX) upon OGR1 over-expression or empty vector. We used transcript expression of CSNK2A1 as control. The result revealed that the presence of PTX abrogated the OGR1 induced up-regulation of expression of CSNK2A3 (Figure 2E, F).

\section{CK2 $\alpha P$ functionally involve in OGR1 induced inhibition of lung cancer migration}

We have previously demonstrated that OGR1 is a metastatic suppressor gene in vitro as well as in vivo using an orthotopic mouse metastasis model [5]. In the current study, we found that OGR1 induces increased expression of CK2 $\alpha \mathrm{P}$ and NEP in transcript and protein levels. Therefore, in the current study, we investigate functional roles of both CK2 $\alpha$ and NEP in the OGR1 induced inhibition of A549 cell migration in vitro using wound healing model in the presence or absence of inhibitor of CK2 $\alpha$ and 
NEP. The results showed that OGR1 inhibits migration of A549 cells. In the presence of CK2 specific inhibitor, CX-4945, the OGR1 induced inhibition of A549 cells migration was completely abrogated (Figure 3A, B). However, thiophan decreases the OGR1 induced inhibition of A549 cell migration only by $\sim 30 \%$, indicating there may be other molecule(s) other than NEP through which CK2 $\alpha \mathrm{P}$ induces inhibition of cancer cell migration following the action of OGR1.

\section{Involvement of Rac1 and cdc42}

The known role of the small GTPase; Rac1 and cdc42 as major drivers of cell motility prompted us to assess whether Rac1 and cdc42 are involved in the OGR1 induced up-regulation of CK2aP expression which leads to inhibition of A549 cells migration. A549 cells were transiently transfected with OGR1 alone or co-transfected with cdcT17N (dominant negative of cdc42) or RacT17N (dominant negative of Rac1). Similarly, control A549 cells were also transiently transfected with empty vector alone and co-transfected with cdcT17N or RacT17N. Initially, over-expression of OGR1 in the OGR1 transfected cells was confirmed by semi-qPCR. The results showed that in the presence of cdcT17N and RacT17N, the OGR1 induced up-regulation of CK2 $\alpha \mathrm{P}$ in A549 cells was significantly abrogated, suggesting that both small G proteins cdc42 and Rac1 are involved in the OGR1 induced up-regulation of CK2 $\alpha \mathrm{P}$ in A549 cells (Figure 4A).

\section{Involvement of MAPK pathway}

The mitogen-activated protein kinase (MAPK) signalling pathway is widely expressed in multicellular organisms, with critical roles in multiple biological processes, such as cell proliferation, death, differentiation, migration, and invasion. To understand the involvement of MAPK pathway in the OGR1 induced up-regulation of CK2aP, selective chemical inhibitors of Jun kinase (JNK), Erk and p38 were treated in the OGR1 or empty vector transfected A549 cells and transcript expression of CK2aP was analysed. The results showed that presence of selective inhibitors of JNK or p38 abrogated the OGR1 induced up-regulation of CK2aP expression whereas presence of selective inhibitor of Erk did not effect on the CK2 $\alpha$ P expression upon OGR1 over-expression (Figure 4B). The overall results indicate that OGR1 inhibits A549 cell migration via up-regulation of CK2 $\alpha$ P and NEP expression through activation of cdc42/Rac1 and MAPK pathways.

\section{Discussion}

Although, OGR1 has been reported as novel metastasis suppressor gene, the molecular mechanism is yet to be understood. In this study, we demonstrate to show that OGR1 regulates expression of a cellular enzyme which acts as a regulator of several hallmarks of cancer cell behaviour CK2 $\alpha$ [16-18] and an important membrane enzyme, neutral endopeptidase 24.11 (NEP, neprilysin, enkephalinase, CD 10). Our finding is the first report, to the best of our knowledge, to demonstrate that the expression of $C S N K 2 A 3(C K 2 \alpha P)$ is regulated by a G protein coupled receptor, OGR1. Our results revealed that 
OGR1 up-regulates the expression CSNK2A3 transcript but not the CSNK2A1 expression. CK2 is ubiquitously expressed and, in contrast it is believed to be a constitutively active enzyme and its regulation appears to be independent of known second messengers [51]. Considering the above findings, the result of immunoblotting against a specific CK2 $\alpha$ antibody which shows up-regulation of CK2 $\alpha$ protein expression when OGR1 is over-expressed in A549 cells indicates that the increased in CK2 $\alpha$ protein may be the translated product of CSNK2A3 (CK2 $\alpha P)$. Therefore, our findings suggest that the aberrantly expressed transcript and/or protein of CK2 $\alpha$ found in various cancer cells may be due to regulated CSNK2A3 $(C K 2 \alpha P)$ expression which is potentially inducible or repressible by several master regulators of developmental pathways. The CSNK2A3 might have an advantage over the CSNK2A1 in cancer cells in which sophisticated lineage specific genes expression is necessary [21]. However, further investigation is necessary to find out the expression of CSNK2A1 and CSNK2A3 in various cancer cells employing strategies which can distinguish expression from one another. It is important to mention that the mRNA sequence of CSNK2A3 is $99.7 \%$ homologous to the CSNK2A3 [21].

Our results further showed OGR1 also up-regulates expression of the transcript as well as protein of NEP, a cell surface cell surface peptidase that is normally expressed by numerous tissues, including prostate, kidney, intestine, endometrium, adrenal glands and lung. Loss or decreases in NEP expression have been reported in a variety of malignancies [52]. Reduced NEP may promote peptide-mediated proliferation by allowing accumulation of higher peptide concentrations at the cell surface, and facilitate the development or progression of neoplasia. It has been shown that the effects of NEP are mediated by its ability to catalytically inactivate substrates such as bombesin and endothelin-1, but also through direct protein-protein interaction with other protein such as Lyn kinase [which associates with the p85 subunit of phosphatidylinositol 3-kinase (PI3-K) resulting in NEP-Lyn-PI3-K protein complex], ezrin/radixin/moesin (ERM) proteins, and the PTEN tumor suppressor protein [52]. To investigate whether CK2 $\alpha \mathrm{P}$ is upstream of NEP or vice-versa in the OGR1 signalling pathway, we analysed the protein expression of CK2 $\alpha$ and NEP using immunoblotting in the presence of DL-thiorphan, a specific NEP inhibitor and silmitasertib (CX-4945), the specific inhibitor for CK2 $\alpha$ respectively in the OGR1 over-expressed or control A549 cells. The result indicated that CK2 $\alpha \mathrm{P}$ is upstream of NEP in the signalling pathway of OGR1; indicating CK2aP induces NEP gene expression as result of OGR1's action. The finding of CK2 $\alpha$ 's regulation of NEP expression is of particular interest because of the role of CK2 $\alpha$ in integrating to essential cellular processes such as cell growth, cell proliferation, cell survival, cell morphology, cell transformation and angiogenesis to proliferation and differentiation signals. In a variety of different cell types, peptide hormones including epidermal growth factor, insulin, and the NEP substrate bombesin increase CK2 $\alpha$ activity. NEP plays a pivotal role in various cancers [40, 41]. It is previously reported CK2 $\alpha$ inhibits NEP activity by phosphorylating cytosolic domain [49]. Therefore, our results reveal another layer of fine-tuning of NEP activity by regulating its expression induced by CK2 $\alpha \mathrm{P}$, the key cellular enzyme.

In our previous study, we revealed that OGR1 inhibits prostate cancer cells migration via constitutive activity of Gai [5]. To investigate whether OGR1's up regulation of CSNK2A3 in A549 is dependent of 
activation of Gai, we analysed the transcript expression of CSNK2A3 in the presence or absence of PTX upon OGR1 over-expression or empty vector. We used transcript expression of CSNK2A1 as control. Our results revealed that OGR1 up-regulate expression of CSNK2A3 transcript via Gai activation.

To find out whether CK2 $\alpha$ P and NEP are functionally involved in the OGR1's induced inhibition of A549 cells, we assessed cell migration using would healing assay in the presence of specific casein kinase $2 \alpha$ inhibitor silmitasertib (CX-4945) or NEP specific inhibitor DL-Thiorphan following overexpression of OGR1 in A549 cells. The result revealed that inhibition of CK2 $\alpha$ abrogates OGR1's induced inhibition of A549 cell migration. The finding strongly suggested OGR1 inhibits cell migration via expression of $\mathrm{CK} 2 \alpha \mathrm{P}$. Our finding supports the previous report that high levels of CK2aP correlate with higher survival rates of lung adenocarcinoma and renal clear cell carcinoma [32, 33]. The result also revealed that inhibitor of NEP abrogates the OGR1's inhibition of A549 cells to some extent (30\%). Our results also revealed that $\mathrm{CK} 2 \mathrm{\alpha P}$ is upstream of NEP and inhibition of casein kinase 2 abrogated completely (100\%) the OGR1 induced inhibition of A549 cell migration whereas inhibition of NEP abrogated only $30 \%$. Therefore, taken together our results suggest there may be other cellular molecule other than NEP to which CK2 $\alpha \mathrm{P}$ targets/activates following OGR1 activation.

To understand molecular mechanism of OGR1 induced up-regulation of CK2aP expression, we assessed roles of small G proteins; Rac1 \& cdc42 and mitogen-activated protein kinases (MAPKs) pathways using negative dominant mutant of small GTPases and specific chemical inhibitors of MAPKs. The result revealed that inhibition of Rac1 and cdc42 activities by respective negative dominant mutant proteins completely abrogated OGR1 induced up-regulation of CSNK2A3 whereas expression of CSNK2A1 is not affected. Cells spread by putting out extensions that contact the surface, form adhesions, and then exert tension to induce outward movement. The process of contact the surface, form adhesions, and then exert tension to induce outward movement are reminiscent of the extensions and adhesions induced by the small GTP-binding proteins Rac and Cdc42 [53].

Further, we also assessed role of MAPKs; extracellular signal-regulated kinase (ERK), Jun kinase (JNK) and p38 since they have been shown to play a key role in transduction extracellular signals to cellular responses. The results clearly showed that inhibition of JNK and p38 completely abrogated OGR1 induced increase expression of CSNK2A3 transcript but inhibition of ERK decreased CSNK2A3 expression some extend only. Taken together, these results indicated that OGR1regulates CK2 $\alpha P$ expression via activation of small GTPase; Rac1 and cdc42 and MAPKs pathways.

The overall findings of this study clearly suggested that OGR1 regulates expression of $C K 2 \alpha P$ and NEP in A549 cells. OGR1 induced up-regulation of CK2 $\alpha P$ through activation of small GTPase; Rac1/cdc42 and JNK/p38 pathways are responsible for inhibition of cancer cell migration. However, OGR1 does not affect the expression of CSNK2A1. There is no previous report of how expression of CK2 $\alpha$ in cancer cells is regulated although many studies have report of aberrant expression of the 
kinase in cancer. Therefore, our findings suggest that the aberrantly expressed transcript and/or protein of CK2 $\alpha$ found in various cancer cells may be due to regulated CSNK2A3/CK2aP expression which is potentially inducible or repressible by several master regulators of developmental pathways. CSNK2A3 might have an advantage over the CSNK2A1 in cancer cells in which sophisticated lineage specific genes expression is necessary.

\section{Material and Methods}

\section{Cell culture and reagents}

The A549 cells (adenocarcinomic human alveolar basal epithelial cells) were procured from the NCCS, Pune, India. The cells were maintained at $37^{\circ} \mathrm{C}, 5 \% \mathrm{CO}_{2}$ in $\mathrm{F}-12 \mathrm{~K}$ Medium (Kaighn's Modification of Ham's F-12 Medium) supplement with 10\% fetal bovine serum (FBS) and 1\% penicillin/streptomycin. All the cell culture media, serum were procured from Gibco, USA while the antibiotics (penicillin/streptomycin) were procured from the Thermo Fisher Scientific (USA). Antibodies for Casein kinase 2 (Santa Cruz Biotechnology), OGR1 Cat No. 72500, abcam), NEP (Cat no. sc-9149, Santacruz Biotechnology) and $\beta$-actin (Cat no. sc-47778, Santa Cruz Biotechnology) were also procured. Specific inhibitors for JNK; SP600125, for p38; SB203580 inhibitors were procured from the abcam while the specific inhibitor for ERK, FR180204, silmitasertib (CX-4945), the specific inhibitor for Casein kinase $2 \alpha$ and DLThiorphan, the specific inhibitor for NEP were also procured from the Sigma-Aldrich.

\section{Plasmid construction and gene transfer}

pcDNA3.1-OGR1 was constructed as described previously [5]. Briefly, the OGR1 coding sequence fragment $(\approx 1.2 \mathrm{~kb}$ ) was amplified and then cloned into pcDNA3.1 (puromycin) by EcoRI and HindllI digestion. The plasmid construct was transformed in $\mathrm{DH} 5 \alpha$ competent $\mathrm{E}$. coli, and selected colonies were then cultured. Plasmid DNA was purified using QuickLyse Miniprep Kit (Qiagen, USA). The construct containing OGR1 was further confirmed by sequencing using the ABI Prism 377 Automated DNA Sequencer (Applied Biosystems, Foster City, CA) and the DNA sequences and reading frames were further verified. The plasmids pcDNA3.1-cdcT17N and pcDNA3.1-RacT17N were procured from Addgene (Cambridge, USA). A549 cells were transiently transfected with 1.0-1.5 $\mathrm{gg}$ plasmids; pcDNA3.1-OGR1 and empty vector (pcDNA3.1) or co-transfected empty vector or pcDNA3.1-OGR1 with pcDNA3.1-cdcT17N and pcDNA3.1-RacT17N using Lipofectamine 2000 (Thermo Fisher Scientific, USA) according to the manufacturer's protocol. The final concentration of the SP600125,

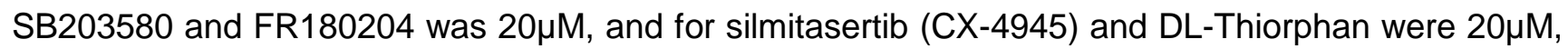
$2.5 \mu \mathrm{M}$ and $10 \mu \mathrm{M}$ respectively. All the inhibitors were added to the cells after $5 \mathrm{hrs}$ of transfection. Treated cells were used for following experiments 48 hours after transfection.

RNA isolation, cDNA synthesis and PCR amplification to measure the expression of casein kinase alpha and neutral endopeptidase 
Total RNAs were extracted from $8 \times 10^{5}$ cultured cells using RNA isolation kit (Qiagen, Germany) according to the manufacturer's instruction. The isolated RNA were analysed for its integrity, purity and yield. Using the isolated RNA as template, first-strand complementary DNA (cDNA) was synthesised using M-MuLV Reverse Transcriptase (NEB, USA). Briefly, the extracted RNA $(\approx 3 \mu \mathrm{g})$ was reverse transcribed in a total volume of $20 \mu \mathrm{l}$ with $350 \mu \mathrm{M}$ dNTP, $50 \mu \mathrm{M}$ oligo(dT), 10X M-MuLV buffer, $200 \mathrm{U}$ RNase inhibitors and 200U M-MuLV reverse transcriptase (NEB). All the reagents were mixed and incubated at $42^{\circ} \mathrm{C}$ for 1 hour followed by $65^{\circ} \mathrm{C}$ for 20 minutes. PCR were then performed using platinum Taq DNA polymerase (TaKara, clontech; Japan). The reaction volume was $50 \mu$, containing 10X PCR buffer, $350 \mu \mathrm{M}$ dNTP mixtures, $0.4 \mathrm{mM}$ of each primer and $5 \mathrm{U}$ Taq DNA polymerase. Cycling conditions were $94^{\circ} \mathrm{C}$ for 4 minutes and then 25 cycles of $\left(94^{\circ} \mathrm{C} 30 \mathrm{~s}, 55^{\circ} \mathrm{C} 30 \mathrm{~s}\right.$, and $\left.72^{\circ} \mathrm{C} 45 \mathrm{~s}\right)$ followed by $72^{\circ} \mathrm{C}$ for 10 minutes. Quantitative PCR were performed with primers obtained from Xcerlis genomics laboratory, Ahmedabad. The primer sequences for OGR1-specific primers; forward primers (5'CTGTCCTGCCAGGTGTGCGG-3') and reverse primers (5'-CACGCGGTGCTGGTTCTCGT-3'). The ß2-microglobulin housekeeping gene (NM_004048) was used as the loading control. The primer sequences used to amplify the $\beta$-microglobulin were (forward) 5'- GAGCCTCGCCTTTGCCGATG-3' and (reverse) 5'-CGATGCCGTGCTCGATGGGG-3'. The primer sequences for CSNK2A1 were (forward) 5'-CCAAACATCAAGTCCAGCTTTGTC-3' and (reverse) 5'-ACCTCGGCCTAATTTTCGAACCA-3' and for CSNK2A3 were (forward) 5'-ATTGCTCCCCACTCCATCGC-3' and (reverse) 5'-ACCTCGGCCTAATTTTCGAACCA-3'. The primer sequences for NEP were (forward) 5'GCCTCTCGGTCCTTGTCCTGC-3'and (reverse) 5'-ACGGGAGCTGGTCTCGGGAA-3'.

\section{Cell lysate preparation and western blotting}

Total cell lysates were prepared with radioimmunoprecipitation assay (RIPA) buffer. Briefly cells were lysed in $300 \mu$ l of RIPA buffer (10 mM Tris- $\mathrm{HCl}$ [pH 7.4], $150 \mathrm{mM} \mathrm{NaCl}, 1 \%$ Triton X-100, $5 \mathrm{mM}$ EDTA, $1 \%$ sodium deoxycholate, $0.1 \%$ SDS, $1.2 \%$ aprotinin, $5 \mu \mathrm{M}$ leupeptin, $4 \mu \mathrm{M}$ antipain, $1 \mathrm{mM}$ phenylmethylsulfonyl fluoride (PMSF), and $0.1 \mathrm{mM} \mathrm{Na3VO4}$, sodium orthovanadate). Cell lysate was then centrifuge at $17,000 \mathrm{xg}$ for $1 \mathrm{hr}$ and supernatant was mixed with $4 \mathrm{X}$ Laemmli sample buffer and equal quantity of protein samples were resolved by SDS-PAGE on $10 \%$ gels and transferred onto PVDF membranes. The membranes were blocked with $5 \%$ non-fat milk for $1 \mathrm{hr}$, incubated with primary antibodies (1:2000 dilution) at $4^{\circ} \mathrm{C}$ overnight and then with secondary antibody (1:6000 dilution) for $1 \mathrm{hr}$ at room temperature. The blots were detected using chemiluminescent ECL system (GE Healthcare). Blot's Images were captured using ChemDoc (BioRad, USA).

\section{Wound healing assay}

To analyse the effect of OGR1 and CK2 $\alpha$, A549 cell was seeded in the six well plates so as to obtain $70-90 \%$ confluency at the time of transfection. After the serum starved for overnight, Cells were transfected with empty vector (pcDNA3.1) or pcDNA3.1-OGR1. After 5 hrs of transfection, cell-free area was created (scratched) in a $70-90 \%$ confluent monolayer cells using $100 \mu$ filter tips. The detached 
bioRxiv preprint doi: https://doi.org/10.1101/556720; this version posted June 11,2019 . The copyright holder for this preprint (which was

not certified by peer review) is the author/funder, who has granted bioRxiv a license to display the preprint in perpetuity. It is made available under aCC-BY-NC-ND 4.0 International license.

cells were removed by washing with $500 \mu \mathrm{L}$ PBS. $2 \mathrm{ml}$ of fresh medium with or without Inhibitors; silmitasertib (CX-4945) and DL-Thiorphan were added afterwards and incubated. Cell migration was observed under the microscope and image was taken at 0-, 12-, 24- and 48- hrs).

\section{Acknowledgements}

We are grateful to the Department of Biotechnology (DBT), Govt. of India and Science and Engineering Research Board, Department of Science and technology (DST-SERB), Govt of India for the research grant to LSS.

\section{Competing Interests}

The authors declare no competing interests.

\section{Funding}

LSS was supported by a research grant (Grant No.: BT/PR15888/NER/95/26/2015 dated 12th January, 2017) from the Department of Biotechnology (DBT), Govt. of India and (Grant No. EMR/2015/001790 dated 17th May 2016) Department of Science and technology (DST-SERB), Govt. of India. The funders had no role in study design, data collection and analysis, decision to publish, or preparation of the manuscript.

\section{Authors' contributions}

ALS, PMM \& NTS performance of the experiments; LSS and ALS analyzed the data and wrote the first draft of manuscript; LSS \& TRS reviewed \& edited the manuscript; LSS supervised the project; LSS acquire the funding. All authors read and approved the final version of the manuscript.

\section{References}

1. Tosa, N., et al., Critical function of T cell death-associated gene 8 in glucocorticoid-induced thymocyte apoptosis. Int Immunol, 2003. 15(6): p. 741-9.

2. Kim, K.S., et al., GPR4 plays a critical role in endothelial cell functions and mediates the effects of sphingosylphosphorylcholine. FASEB J, 2005. 19(7): p. 819-21. 
3. Radu, C.G., A. Nijagal, J. McLaughlin, L. Wang and O.N. Witte, Differential proton sensitivity of related $G$ protein-coupled receptors T cell death-associated gene 8 and G2A expressed in immune cells. Proc Natl Acad Sci U S A, 2005. 102(5): p. 1632-7.

4. Qiao, J., et al., Lysophosphatidylcholine impairs endothelial barrier function through the G protein-coupled receptor GPR4. Am J Physiol Lung Cell Mol Physiol, 2006. 291(1): p. L91-101.

5. Singh, L.S., et al., Ovarian cancer G protein-coupled receptor 1, a new metastasis suppressor gene in prostate cancer. J Natl Cancer Inst, 2007. 99(17): p. 1313-27.

6. LaTulippe, E., et al., Comprehensive gene expression analysis of prostate cancer reveals distinct transcriptional programs associated with metastatic disease. Cancer Res, 2002. 62(15): p. 4499506.

7. Ren, J. and L. Zhang, Effects of ovarian cancer G protein coupled receptor 1 on the proliferation, migration, and adhesion of human ovarian cancer cells. Chin Med J (Engl), 2011. 124(9): p. 1327-32.

8. Li, J., et al., Ovarian cancer G protein coupled receptor 1 suppresses cell migration of MCF7 breast cancer cells via a Galpha12/13-Rho-Rac1 pathway. J Mol Signal, 2013. 8(1): p. 6.

9. Yan, L., L.S. Singh, L. Zhang and Y. Xu, Role of OGR1 in myeloid-derived cells in prostate cancer. Oncogene,2014. 33(2): p. 157-64.

10. $\mathrm{Li}, \mathrm{H}$., et al., Abnormalities in osteoclastogenesis and decreased tumorigenesis in mice deficient for ovarian cancer G protein-coupled receptor 1. PLoS One, 2009. 4(5): p. e5705.

11. Horman, S.R., et al., Functional profiling of microtumors to identify cancer associated fibroblastderived drug targets. Oncotarget, 2017. 8(59): p. 99913-99930.

12. Ludwig, M.G., et al., Proton-sensing G-protein-coupled receptors. Nature, 2003. 425(6953): p. 93-8.

13. Tomura, H., C. Mogi, K. Sato and F. Okajima, Proton-sensing and lysolipid-sensitive G-proteincoupled receptors: a novel type of multi-functional receptors. Cell Signal, 2005. 17(12): p. 146676.

14. Radu, C.G., et al., Normal immune development and glucocorticoid-induced thymocyte apoptosis in mice deficient for the T-cell death-associated gene 8 receptor. Mol Cell Biol, 2006. 26(2): p. 668-77.

15. Yang, L.V., et al., Vascular abnormalities in mice deficient for the G protein-coupled receptor GPR4 that functions as a pH sensor. Mol Cell Biol, 2007. 27(4): p. 1334-47.

16. Trembley, J.H., et al., Emergence of protein kinase CK2 as a key target in cancer therapy. Biofactors, 2010. 36(3): p. 187-95.

17. Hanahan, D. and R.A. Weinberg, Hallmarks of cancer: the next generation. Cell, 2011. 144(5): p. 646-74.

18. Buontempo, F., et al., Therapeutic targeting of CK2 in acute and chronic leukemias. Leukemia, 2018. 32(1): p. 1-10.

19. Wirkner, U., et al., Human casein kinase II subunit alpha: sequence of a processed (pseudo)gene and its localization on chromosome 11. Biochim Biophys Acta, 1992. 1131(2): p. 220-2. 
20. Devilat, I. and P. Carvallo, Structure and sequence of an intronless gene for human casein kinase II-alpha subunit. FEBS Lett, 1993. 316(2): p. 114-8.

21. Singh, L.S. and M. Kalafatis, Sequencing of full-length cDNA encoding the alpha and beta subunits of human casein kinase II from human platelets and megakaryocytic cells. Expression of the casein kinase Ilalpha intronless gene in a megakaryocytic cell line. Biochemistry, 2002. 41(28): p. 8935-40.

22. Hung, M.S., et al., Functional polymorphism of the CK2alpha intronless gene plays oncogenic roles in lung cancer. PLoS One, 2010. 5(7): p. e11418.

23. Tawfic, S., et al., Protein kinase CK2 signal in neoplasia. Histol Histopathol, 2001. 16(2): p. 57382.

24. Chua, M.M.J., M. Lee and I. Dominguez, Cancer-type dependent expression of CK2 transcripts. PLoS One, 2017. 12(12): p. e0188854.

25. Nurmio, M., et al., Receptor tyrosine kinase inhibition causes simultaneous bone loss and excess bone formation within growing bone in rats. Toxicol Appl Pharmacol, 2011. 254(3): p. 267-79.

26. Peng, B., et al., Modulation of LSD1 phosphorylation by CK2/WIP1 regulates RNF168-dependent 53BP1 recruitment in response to DNA damage. Nucleic Acids Res, 2015. 43(12): p. 5936-47.

27. Bachmanov, A.A., D.R. Reed, G.K. Beauchamp and M.G. Tordoff, Food intake, water intake, and drinking spout side preference of 28 mouse strains. Behav Genet, 2002. 32(6): p. 435-43.

28. Champagne, M.A., et al., Imatinib mesylate (STI571) for treatment of children with Philadelphia chromosome-positive leukemia: results from a Children's Oncology Group phase 1 study. Blood, 2004. 104(9): p. 2655-60.

29. Vandyke, K., et al., Imatinib mesylate causes growth plate closure in vivo. Leukemia, 2009. 23(11): p. 2155-9.

30. Russo, M., et al., CK2 and PI3K are direct molecular targets of quercetin in chronic lymphocytic leukaemia. Oncotarget, 2017. 8(26): p. 42571-42587.

31. Picard, S., et al., Trough imatinib plasma levels are associated with both cytogenetic and molecular responses to standard-dose imatinib in chronic myeloid leukemia. Blood, 2007. 109(8): p. 3496-9.

32. Chua, M.M., et al., CK2 in Cancer: Cellular and Biochemical Mechanisms and Potential Therapeutic Target. Pharmaceuticals (Basel), 2017. 10(1).

33. Ortega, C.E., Y. Seidner and I. Dominguez, Mining CK2 in cancer. PLoS One, 2014. 9(12): p. e115609.

34. Das, N., N. Datta, U. Chatterjee and M.K. Ghosh, Estrogen receptor alpha transcriptionally activates casein kinase 2 alpha: A pivotal regulator of promyelocytic leukaemia protein (PML) and AKT in oncogenesis. Cell Signal, 2016. 28(6): p. 675-87.

35. Kim, H.S., et al., Oncogenic potential of CK2alpha and its regulatory role in EGF-induced HDAC2 expression in human liver cancer. FEBS J, 2014. 281(3): p. 851-61.

36. Nitta, R.T., et al., Casein kinase 2alpha regulates glioblastoma brain tumor-initiating cell growth through the beta-catenin pathway. Oncogene, 2015. 34(28): p. 3688-99. 
37. Rabjerg, M., et al., Molecular characterization of clear cell renal cell carcinoma identifies CSNK2A1, SPP1 and DEFB1 as promising novel prognostic markers. APMIS, 2016. 124(5): p. 372-83.

38. Sumitomo, M., R. Shen and D.M. Nanus, Involvement of neutral endopeptidase in neoplastic progression. Biochim Biophys Acta, 2005. 1751(1): p. 52-9.

39. Shipp, M.A., et al., CD10/neutral endopeptidase 24.11 hydrolyzes bombesin-like peptides and regulates the growth of small cell carcinomas of the lung. Proc Natl Acad Sci U S A, 1991. 88(23): p. 10662-6.

40. Shipp, M.A. and A.T. Look, Hematopoietic differentiation antigens that are membrane-associated enzymes: cutting is the key! Blood, 1993. 82(4): p. 1052-70.

41. Ganju, R.K., R.G. Shpektor, D.G. Brenner and M.A. Shipp, CD10/neutral endopeptidase 24.11 is phosphorylated by casein kinase II and coassociates with other phosphoproteins including the lyn src-related kinase. Blood, 1996. 88(11): p. 4159-65.

42. Angelisova, P., K. Drbal, V. Horejsi and J. Cerny, Association of CD10/neutral endopeptidase 24.11 with membrane microdomains rich in glycosylphosphatidylinositol-anchored proteins and Lyn kinase. Blood, 1999. 93(4): p. 1437-9.

43. Cutrona, G., et al., Expression of CD10 by human T cells that undergo apoptosis both in vitro and in vivo. Blood, 1999. 94(9): p. 3067-76.

44. Tokuhara, T., et al., Neutral endopeptidase/CD10 and aminopeptidase N/CD13 gene expression as a prognostic factor in non-small cell lung cancer. Jpn J Thorac Cardiovasc Surg, 2001. 49(8): p. 489-96.

45. Gurel, D., A. Kargi, I. Karaman, A. Onen and M. Unlu, CD10 expression in epithelial and stromal cells of non-small cell lung carcinoma (NSCLC): a clinic and pathologic correlation. Pathol Oncol Res, 2012. 18(2): p. 153-60.

46. Kristiansen, G., K. Schluns, Y. Yongwei, M. Dietel and I. Petersen, CD10 expression in non-small cell lung cancer. Anal Cell Pathol, 2002. 24(1): p. 41-6.

47. Ono, S., et al., Podoplanin-positive cancer-associated fibroblasts could have prognostic value independent of cancer cell phenotype in stage I lung squamous cell carcinoma: usefulness of combining analysis of both cancer cell phenotype and cancer-associated fibroblast phenotype. Chest, 2013. 143(4): p. 963-970.

48. Leithner, K., et al., Hypoxia increases membrane metallo-endopeptidase expression in a novel lung cancer ex vivo model - role of tumor stroma cells. BMC Cancer, 2014. 14: p. 40.

49. Siepmann, M., S. Kumar, G. Mayer and J. Walter, Casein kinase 2 dependent phosphorylation of neprilysin regulates receptor tyrosine kinase signaling to Akt. PLoS One, 2010. 5(10).

50. Mandal, T., et al., Reduced phosphorylation of Stat3 at Ser-727 mediated by casein kinase 2 protein phosphatase 2A enhances Stat3 Tyr-705 induced tumorigenic potential of glioma cells. Cell Signal, 2014. 26(8): p. 1725-34.

51. Guerra, B., et al., CK2: a protein kinase in need of control. Pharmacol Ther, 1999. 82(2-3): p. 303-13. 
52. Sumitomo, M., et al., Neutral endopeptidase inhibits prostate cancer cell migration by blocking focal adhesion kinase signaling. J Clin Invest, 2000. 106(11): p. 1399-407.

53. Price, L.S., J. Leng, M.A. Schwartz and G.M. Bokoch, Activation of Rac and Cdc42 by integrins mediates cell spreading. Mol Biol Cell, 1998. 9(7): p. 1863-71.

\section{Figure legends}

Figure 1: OGR1 up-regulates CSNK2A3 (CK2 $\alpha$ intronless gene) and NEP but not CSNK2A1 in A549: (A) A549 cells were transfected with OGR1 (OGR1) or pcDNA3.1 (Vector), transcript expressions of CSNK2A1, CSNK2A3 and NEP were analysed in the by semi-quantitative RT-PCR. $\beta$-actin was used as control for equal loading. (B) RNA bands intensities for CSNK2A1 and CSNK2A3 were analysed by using Image Acquisition and Analysis software (BioRad, USA) and presented in graph. (C) Protein expressions of NEP and CK2 $\alpha$ were assayed by Western blotting with specific antibodies and $\beta$-actin was used as control for equal landing, (D) protein band intensities were analysed by using Image Acquisition and Analysis software (BioRad, USA) and presented as graph. Experiments were repeated thrice. Statistical significance between control (Vector) and OGR1 was calculated using student's "t" test. Bars indicates SD, ** indicates $p$-value $<0.01$ and ${ }^{* * *}$ indicates $p$-value $<0.001$.

Figure 2: CK2 is upstream of NEP in the OGR1 signalling pathway: Western blot assay of NEP and CK2 $\alpha$ expression in presence or absence of specific inhibitors. (A) A549 cells were transfected with pDNA3.1-OGR1 (OGR1) or pcDNA3.1 (Vector) and NEP expression was analysed with anti NEP in presence or absence of CX-4945, CK2 inhibitor (CX4945) and (B) protein band intensities were analysed by using Image Acquisition and Analysis software (BioRad, USA) and presented as graph, (C) expression of CK2 $\alpha$ was analysed with anti CK2 in presence or absence of NEP inhibitor, thiophan (THP) and (D) protein band intensities were analysed by using Image Acquisition and Analysis software (BioRad, USA) and presented as graph, (E) Semi Quantitaive PCR showing the expression of NEP and CK2 in presence or absence of Gai inhibitor PTX and (F) intensities of protein bands were analysed by using Image Acquisition and Analysis software (BioRad, USA) and presented in graph. Statistical significance between treated and untreated was calculated using student's "t" test. Bars indicate $\mathrm{SD},{ }^{*}$ indicates $p$-value $<0.04,{ }^{* *}$ indicates $p$-value $<0.01$ and ${ }^{* * *}$ indicates $p$-value $<0.001$.

Figure 3: OGR1 inhibits A549 cell migration via CK2: (A) OGR1 inhibits A549 cell migration but presence of CK2 inhibitor, CX4945 abrogated the effect of OGR1 but not the presence of NEP inhibitor, thiophan (THP). A549 cell were transfected with pDNA3.1-OGR1 or pcDNA3.1 (Vector) in presence of CK2 and NEP inhibitors. Cell migration was analysed and image was taken hourly. (B) To measure the area of wound, the length of scratches were measured on the light microscope and plotted on graph. Wound area of control $0 \mathrm{hr}$ was taken as $100 \%$ and means \& SD. P-values were calculated comparing with the control values of the same hrs of treatment by students' " $t$ " test. The bar (Red) indicates the scale of $100 \mu \mathrm{m}$. Bars indicate SD, * indicates $p$-value $<0.04$, ${ }^{* *}$ indicates $p$-value $<0.01$ and ${ }^{* * *}$ indicates $p$-value $<0.001$. 
bioRxiv preprint doi: https://doi.org/10.1101/556720; this version posted June 11,2019 . The copyright holder for this preprint (which was

not certified by peer review) is the author/funder, who has granted bioRxiv a license to display the preprint in perpetuity. It is made available under aCC-BY-NC-ND 4.0 International license.

Figure 4: Role of Rac/CDC42 and MAPK pathways in regulation of CK2 $\alpha$ genes induced by

OGR1: (A) A549 cells were co-transfected with OGR1 or pcDNA3.1 (Vector) and negative dominant mutants of Rac (pcDNA3.1-RacT17N) or negative dominant mutants of CDC42 (pcDNA3.1-cdcT17N). Semi-quantitative RT-PCR of was performed to analyse CSNK2A1 and CSNK2A3 transcript expression. $\beta$-actin was used as control for equal loading; Inhibition of Rac activity abrogated up-regulation of CSNK2A3 induced by OGR1 (B) A549 cells were also transfected with pDNA3.1-OGR1 or pcDNA3.1 (Vector) in presence of MAPK kinase inhibitors. Semi-quantitative RT-PCR of was performed to analyse CSNK2A1 and CSNK2A3 transcript expression. $\beta$-actin was used as control for equal loading; inhibition of JNK and p38 using specific inhibitors, SP600125 and SB2203580 respectively, abrogated the up-regulate of CSNK2A3 induced by OGR1 but not by inhibition of ERK (FR180204) whereas inhibition of ERK, JNK and p38 does not significantly affect the expression of CSNK2A1 in A549.

Figure 5 Speculative signaling pathways of OGR1 for inhibition of A549 cell migration is illustrated. OGR1 activates protein Gai which leads to further activation of small G proteins Rac1 and cdc42. Activations of Rac1 and cdc42 lead to activation of MAPK pathways which further increase expression of CK2 $\alpha \mathrm{P}$ and one or more other proteins (?), and finically inhibit cell migration. 
Figure 1

(A)

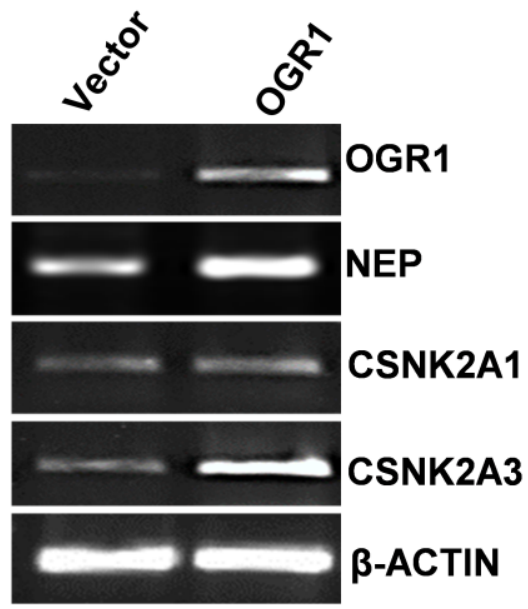

(C)

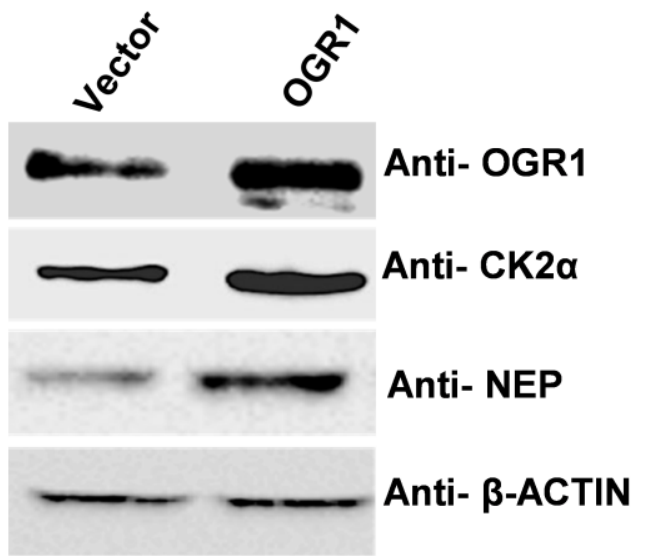

(B)

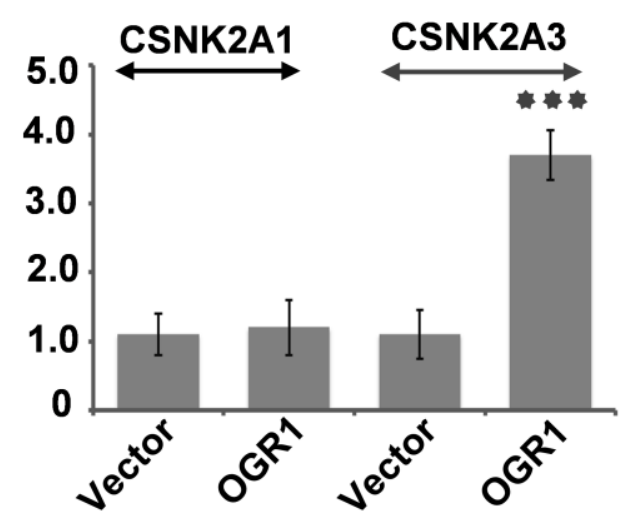

(D)

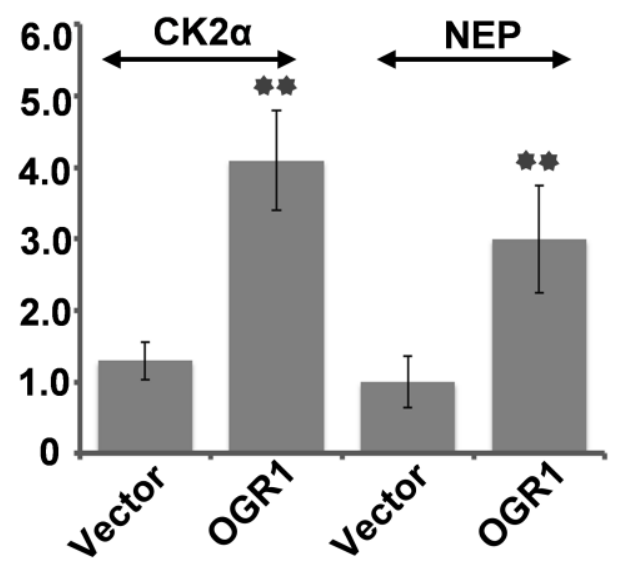


Figure 2

(A)

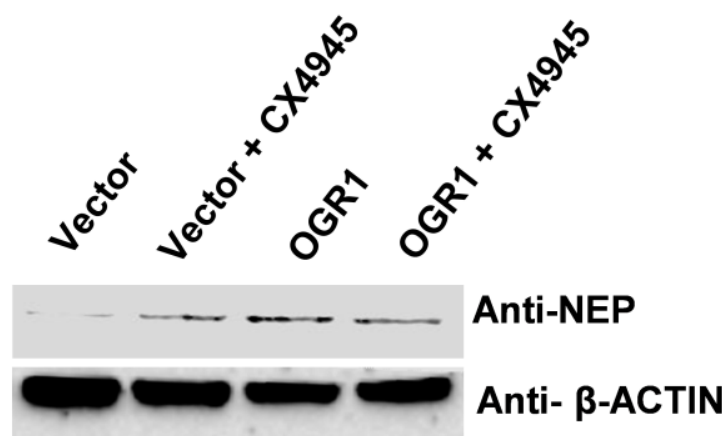

(C)

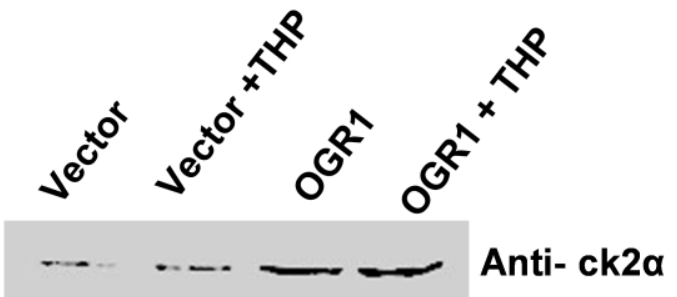

(E)
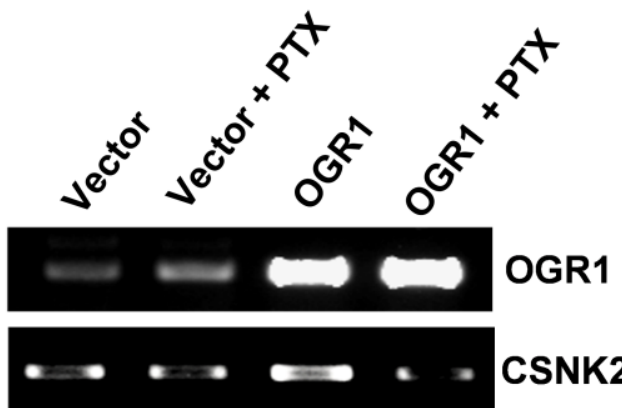

CSNK2A3

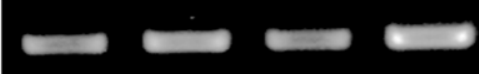

CSNK2A1
(B)

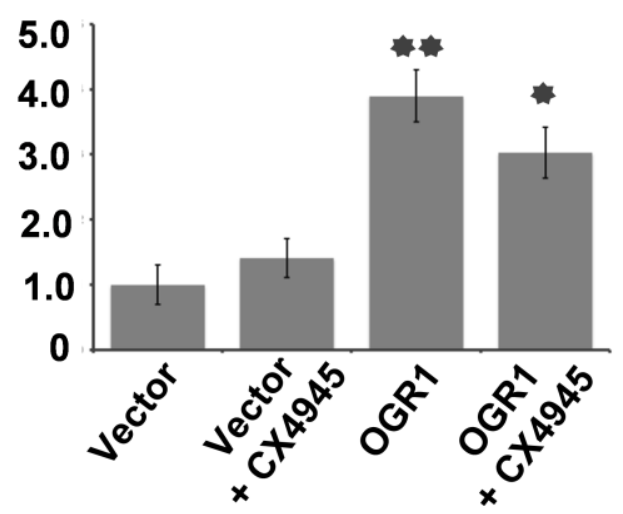

(D)

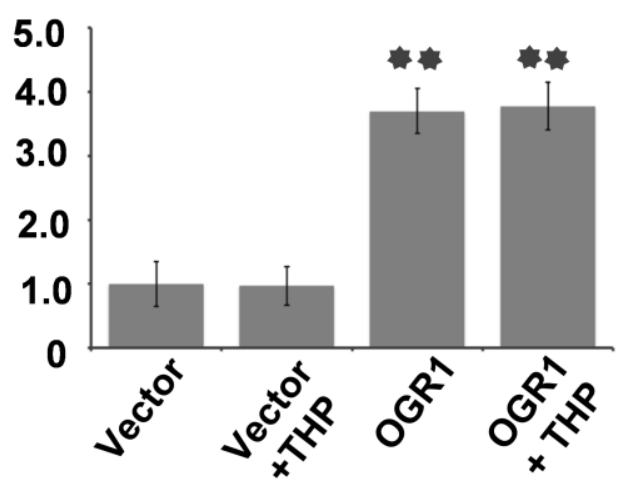

(F)

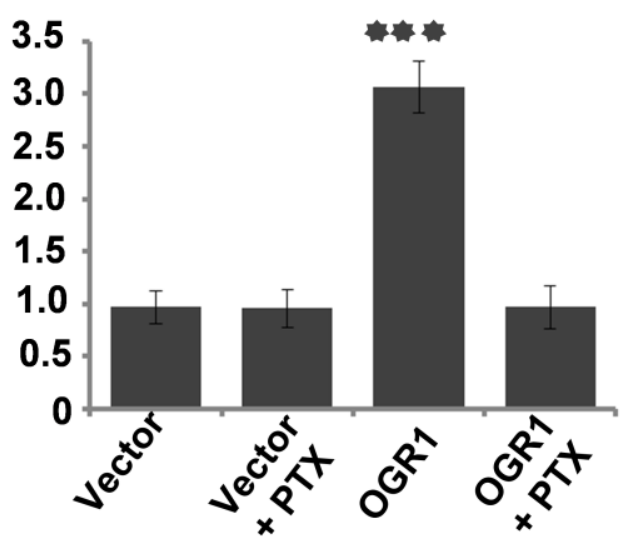


Figure 3

(A)

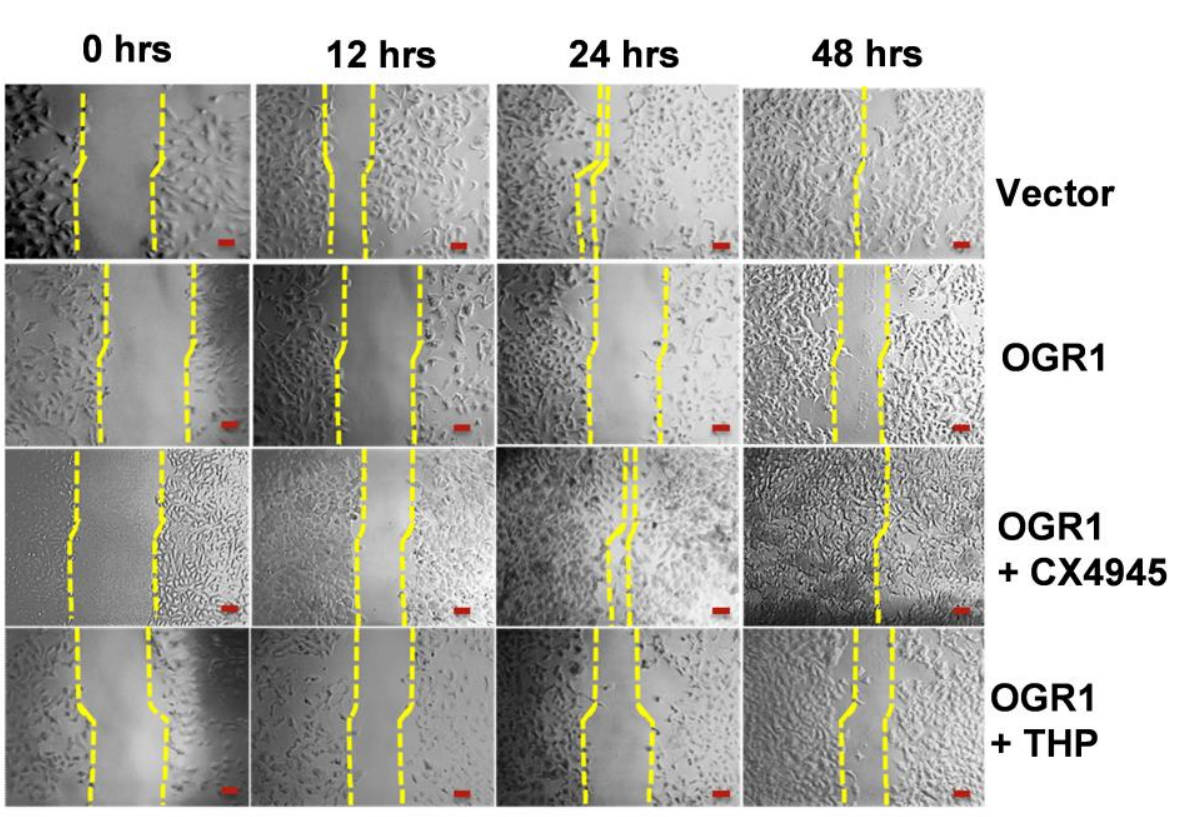

(B)

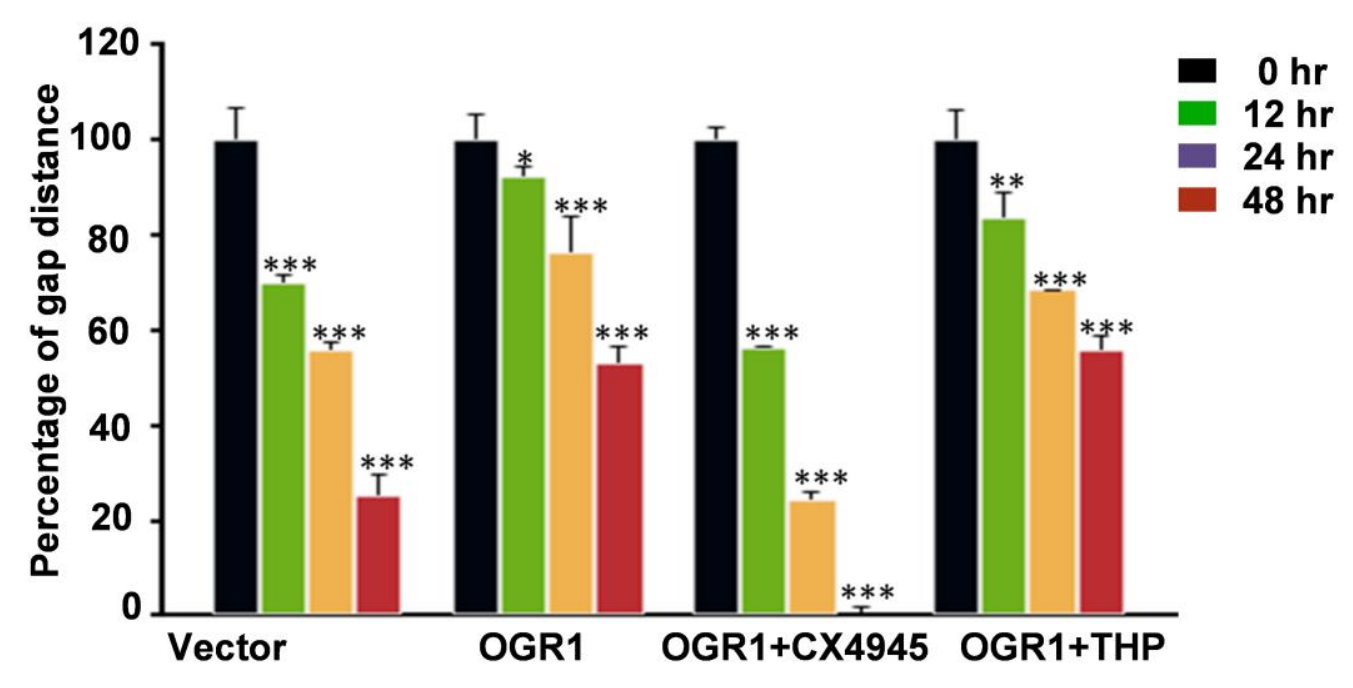


Figure 4

(A)

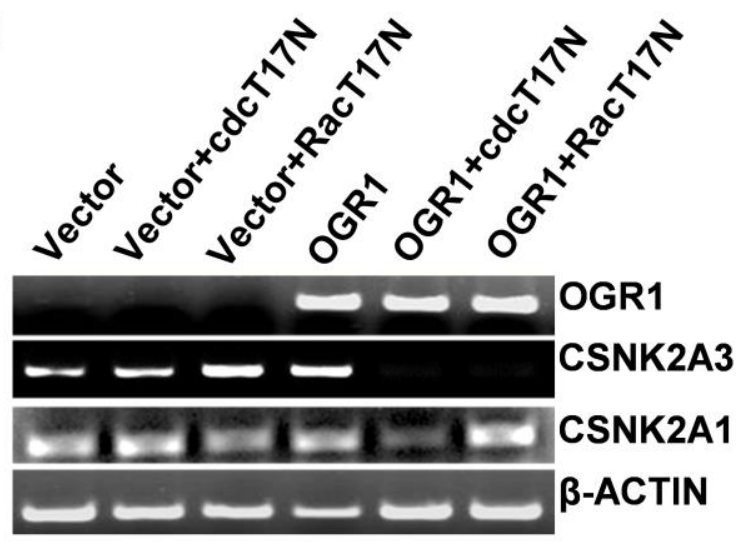

(B)

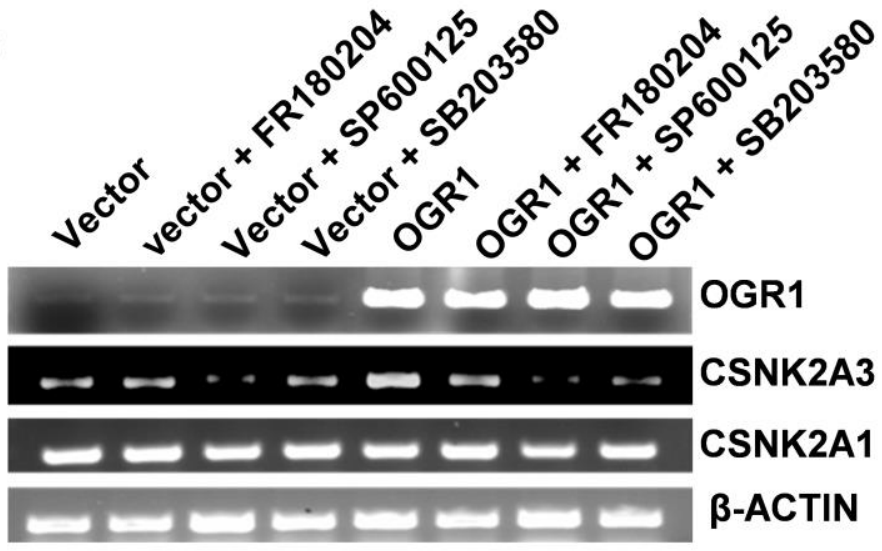


bioRxiv preprint doi: https://doi.org/10.1101/556720; this version posted June 11,2019 . The copyright holder for this preprint (which was

not certified by peer review) is the author/funder, who has granted bioRxiv a license to display the preprint in perpetuity. It is made available under aCC-BY-NC-ND 4.0 International license.

Figure 5
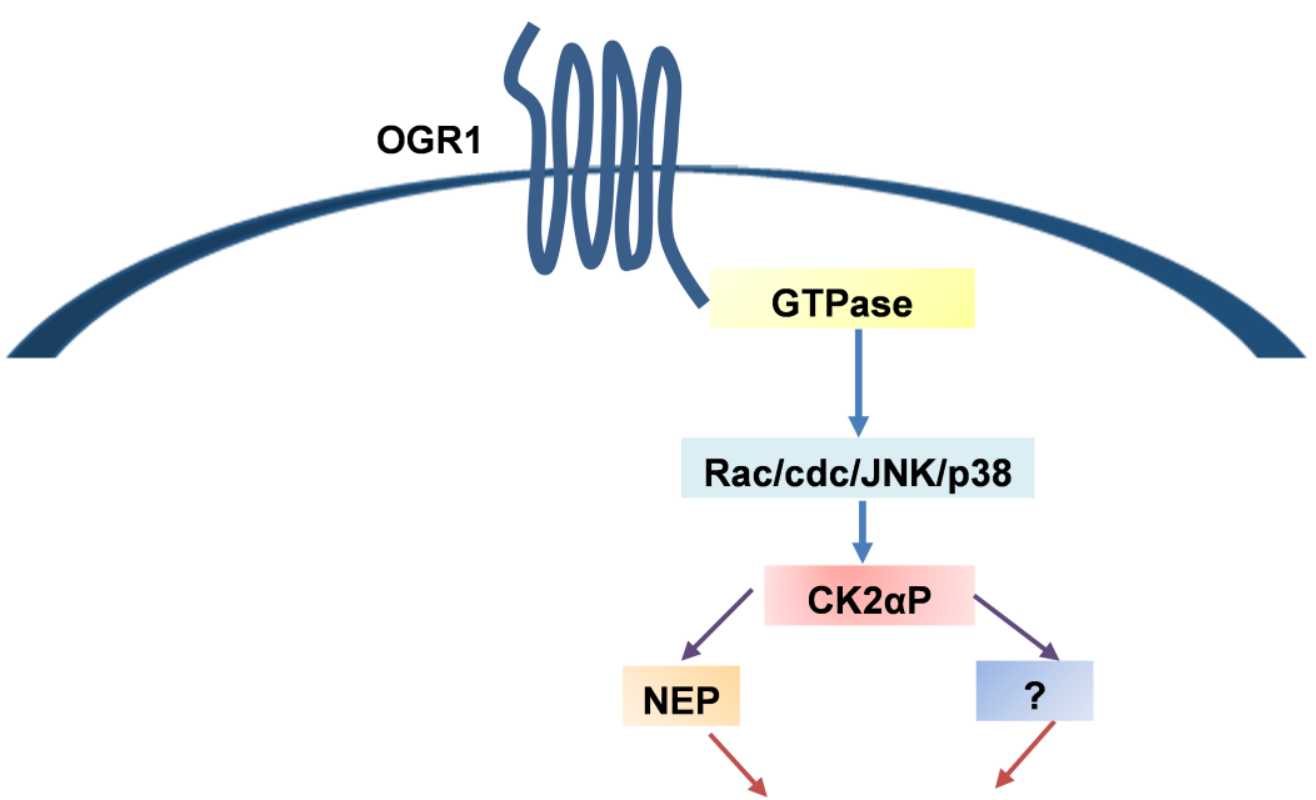

Cell movement

inhibition of cancer cell migration 